\title{
Statistical properties of frequency shifted feedback lasers
}

\author{
H. Guillet de Chatellus, J.-P. Pique, \\ Laboratoire de Spectrométrie Physique, \\ UMR 5588 CNRS-Université Joseph Fourier, \\ 140 Av. de la Physique, \\ BP 87 - 38402 Saint Martin d'Hères, France
}

February 24, 2014

\begin{abstract}
We evidence experimentally the statistical properties of frequency shifted feedback (FSF) lasers through measurements of the homodyne beat signal and interferometric autocorrelation of a dye FSF laser at the output of a Michelson interferometer. The FSF laser is found to show thermal fluctuations and photon bunching. Moreover whereas the degree of first-order coherence vanishes beyond the coherence length of the FSF source, the degree of second-order coherence exhibits periodic revivals far beyond the coherence length, with a period equal to the cavity roundtrip time. Our observations are in good agreement with the theoretical treatment of Yatsenko et al. (Opt. Comm. 282 (2009) 300) and validate the description of the output field of a FSF laser by a broadband cyclostationary thermal field.
\end{abstract}

\section{Introduction and methods}

Frequency shifted feedback (FSF) lasers constitute remarkable light sources, in terms of both practical applications and fundamental research. A FSF laser is a gain cavity in which an acousto-optics (AO) frequency shifter is inserted. Each time a photon makes a roundtrip in the cavity, it experiences a frequency shift, usually in the range of a few tens of $\mathrm{MHz}$ [1]. If the frequency shift is commensurable with the cavity free spectral range, the FSF laser emits in the pulsed mode [2]. Otherwise the output field is CW, the interferences responsible for the usual cavity comb spectrum are washed out and the resulting output spectrum is continuous [3]. Its width is adjustable by means of intracavity selective elements and can be tailored by seeding the FSF cavity with an external laser [4]. The broadband spectrum finds naturally applications in atomic physics since it enables to excite a whole Doppler-hyperfine transition [5]. Optical pumping of all velocity classes of an atomic vapor has recently being 
applied to high resolution interferometry [6]. Since it optimizes the excitation of an atomic transition by reducing the saturation, FSF lasers are also currently implemented in the field of laser guide stars for astronomy (LGS) where one seeks to excite as efficiently as possible the mesospheric sodium to use the return fluorescence as an intense artificial guide star to pilot the adaptive optics loop $[7,8]$. Other applications have also been demonstrated in white light cooling of atoms [9] and laser induced atomic drift [10]. But so far, most of the applications of FSF lasers concern metrology, where their dynamical properties have led to promising implementations. Namely when the FSF laser beam is split in an interferometer and recombined, a RF modulation is observed in the homodyne signal and the beat frequency is proportional to the path difference of the interferometer. This result is valid far beyond the coherence length of the laser source and demonstrates the intrinsic chirped nature of the FSF laser field. Applications to optical frequency domain ranging [11, 12, 13], measurement of group velocity dispersion in fibers [14], vibrometry [15], temperature measurement [16] have recently been demonstrated. Other applications also include multiple access optical communications [17].

The puzzling behaviour of FSF sources have lead to multiple studies on the dynamics of such lasers $[18,19,20,21]$, and to some controversy on the nature of the light field, in particular whether the chirp was continuous or not $[22,23,24]$. A simple and intuitive image of the output of the FSF laser has emerged, consisting in a comb of modes chirping with time, at a rate proportional to the intracavity frequency shift $[11,25,26,27]$. This description enables to explain easily the homodyne properties of FSF lasers. However the question of the origin of this frequency comb remained unanswered. Recently a theoretical study showed that there was no need to invoke the description by the chirping comb since homodyne RF properties can be deduced from the intuitive picture, where the output field is cyclostationary and consists in an infinite sequence of spontaneous emission events emitted in the gain curve of the cavity. Each of them repeats periodically at the cavity roundtrip time, experiencing a periodical frequency shift [28]. This model succeeds remarkably in explaining the RF properties of the FSF laser field while being compatible with earlier experimental studies [29]: heterodyne measurements in particular had shown that the broadband nature of the FSF field relied mostly on chaotic (white) phase fluctuations, which is consistent with the role played by spontaneous emission. Contrary to the phase noise, intensity noise of a FSF laser (which can be directly measured on the RF spectrum of the laser output) shows usually discrete beats at integer multiples of the free spectral range (FSR), and also additional noise induced by the frequency shifter. In some cases however FSF sources showed no repetitive intensity modulation at the laser FSR [30,31]. But in any case no study has been done yet to quantify the intensity fluctuations of FSF lasers and to link them to their statistical properties.

In this paper we investigate both theoretically and experimentally the statistical properties of a dye FSF laser by characterizing the degree of second-order coherence (or the intensity correlation function) $g^{(2)}(\tau)$. Since the coherence time of our laser is in the ps range, measuring photoelectrons statistics during 
a variable observation time is not appropriate in our case [32]. Instead we use the technique of second-harmonic generation (SHG) whose efficiency is directly linked to the intensity fluctuations of the laser source [33, 34]. To access the second-order coherence function we adopt an interferometric autocorrelation (IAC) scheme $[35,36]$ where the laser beam is split in two arms experiencing a variable delay $\tau$ and recombined in the same spatial mode into a type I nonlinear crystal tuned to the phase-matching condition for SHG. The intensity correlation function can be deduced from the variation of the harmonic signal with the path difference.

In the first part of the paper we investigate theoretically the degrees of firstand second-order coherence $g^{(1)}(\tau)$ and $g^{(2)}(\tau)$ of FSF lasers using the passive frequency-independent cavity model given in [28] and calculate the interferometric autocorrelation function of a FSF laser, compared to a chaotic light source with the same spectral width. Thermal photon statistics and a periodic behavior of $g^{(2)}(\tau)$ are predicted.

Then in the second part we describe the experimental measurement of the second-order coherence function through measurement of the autocorrelation function of a dye FSF laser at the output of a Michelson interferometer. Our measurements confirm both the thermal statistics (i.e. the photon bunching) of the FSF laser and the periodic nature of the degree of second-order coherence beyond the (first-order) coherence domain. Finally novel applications of FSF lasers exploiting their coherence properties are proposed.

\section{Theoretical description}

\subsection{Chaotic light}

First we briefly review elementary aspects of the coherence properties of chaotic light fields: this point is useful for further comparison to the output field of a FSF laser.

We consider $\xi(t)$ a stationary chaotic light field in a defined spatial mode, generating the time-dependent intensity $I(t)=\xi(t) \xi(t)^{*}$.

For an ergodic process the time-average intensity $I_{0}=\overline{I(t)}$ equals the ensemble-average $\langle I(t)\rangle$. We also assume a Lorentzian field with a spectral density given by:

$$
\tilde{I}(\omega)=\frac{\gamma / \pi}{\left(\omega_{0}-\omega\right)^{2}+\gamma^{2}} I_{0}
$$

where $\gamma$ and $\omega_{0}$ characterize respectively the width and the central angular frequency of the spectral distribution of this Lorentzian chaotic (LC) field [37]. The corresponding correlation function of the field is:

$$
F(\tau)=\left\langle\xi(t) \xi^{*}(t+\tau)\right\rangle=I_{0} e^{-i \omega_{0} \tau-\gamma|\tau|} .
$$

The degrees of first- and second-order coherence are respectively: 


$$
g_{L C}^{(1)}(\tau)=\frac{\left\langle\xi(t) \xi^{*}(t+\tau)\right\rangle}{\left\langle\xi(t) \xi^{*}(t)\right\rangle}=e^{-i \omega_{0} \tau-\gamma|\tau|}
$$

and

$$
g_{L C}^{(2)}(\tau)=\frac{\left\langle\xi(t) \xi^{*}(t) \xi(t+\tau) \xi^{*}(t+\tau)\right\rangle}{\left\langle\xi(t) \xi^{*}(t)\right\rangle^{2}}=1+e^{-2 \gamma|\tau|}
$$

where we use the factorization rule valid for chaotic fields:

$$
\begin{aligned}
\left\langle\xi\left(t_{1}\right) \xi^{*}\left(t_{2}\right) \xi\left(t_{3}\right) \xi^{*}\left(t_{4}\right)\right\rangle & =\left\langle\xi\left(t_{1}\right) \xi^{*}\left(t_{2}\right)\right\rangle\left\langle\xi\left(t_{3}\right) \xi^{*}\left(t_{4}\right)\right\rangle \\
& +\left\langle\xi\left(t_{1}\right) \xi^{*}\left(t_{4}\right)\right\rangle\left\langle\xi\left(t_{3}\right) \xi^{*}\left(t_{2}\right)\right\rangle .
\end{aligned}
$$

Then a chaotic light source exhibits the usual properties of thermal fluctuations or photon bunching $g^{(2)}(0)=2$. In other words the variance of the process defined as $\sigma^{2}=\left\langle I(t)^{2}\right\rangle-\langle I(t)\rangle^{2}$ is equal to $\langle I(t)\rangle^{2}$ : intensity fluctuations of the chaotic field are comparable to the mean intensity. The degrees of first- and second-order coherence of the LC light source are plotted on fig. 1 (top).

We now turn to the case where the previous LC field feeds a balanced interferometer, with a time delay $\tau$ between both arms. At the output of the interferometer a non-linear crystal performs the interferometric autocorrelation (IAC) function of the light field. to:

The time-dependent intensity of the second-harmonic field is proportional

$$
I_{L C}^{I A C}(t, \tau)=1 / 16\left|(\xi(t)+\xi(t+\tau))^{2}\right|^{2} .
$$

After simple calculations the time-average harmonic intensity is:

$$
\begin{aligned}
& I_{L C}^{I A C}(\tau)=\overline{I_{L C}^{I A C}(t, \tau)}= \\
& \frac{I_{0}^{2}}{4}\left[2+4 e^{-\gamma|\tau|} \cos \left(\omega_{0} \tau\right)+e^{-2 \gamma|\tau|} \cos \left(2 \omega_{0} \tau\right)+e^{-2 \gamma|\tau|}\right] .
\end{aligned}
$$

The interferometric autocorrelation trace is plotted on fig. 1 (bottom). The ratio of the maximum at $\tau=0$ to the background is $8: 2$, and $3: 2$ when averaging is performed over the fringes.

\subsection{Passive frequency-independent FSF cavity}

In this paragraph we turn to the statistical properties of the FSF laser field: we assume that the light field results from the intracavity spontaneous emission and consists in periodic trains of chaotic Lorentzian light shifted in frequency at each roundtrip (cyclostationary process). We consider the simple model of a passive frequency independent cavity with no gain and whose losses are simply due to the reflection on the weakly transmitting output mirror, defined by a reflection 

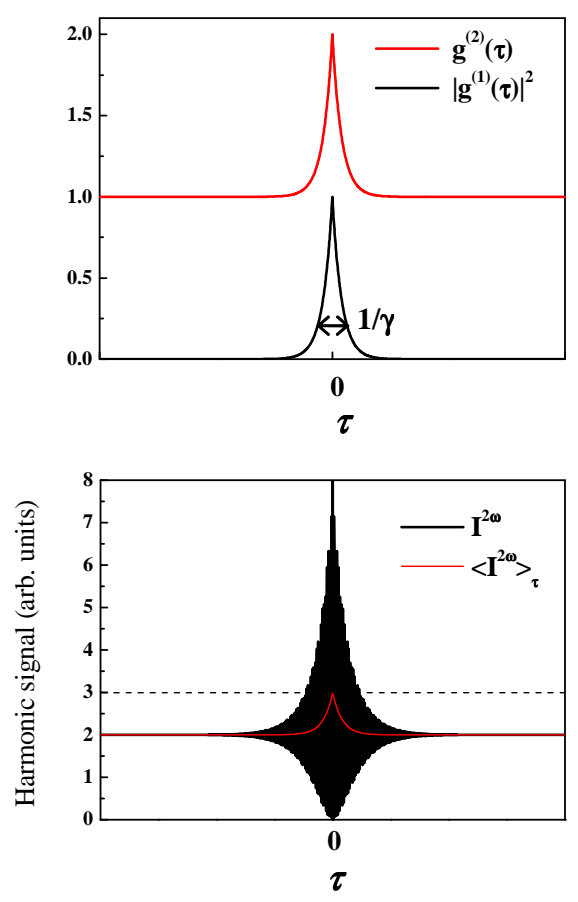

Figure 1: Top: plot of the $\left|g^{(1)}(\tau)\right|^{2}$ and $g^{(2)}(\tau)$ functions of the LC light field. Bottom: interferometric autocorrelation trace of the LC light field without (black) and with an average over the fringes (red). 
coefficient $R(R \lesssim 1)$. For the sake of clarity we take the same notations as [28]: $\Delta$ is the angular frequency shift experienced by the cavity photons per roundtrip (in the case of a linear cavity, twice the AO angular frequency), $\tau_{r}$ is the roundtrip time in the cavity. Considering that the field originates solely from spontaneous emission described by a chaotic field $\xi(t)$, the electric field at the output of the laser cavity is proportional to the intracavity laser field, which can be defined by an infinite sum [28]:

$$
E(t)=\sum_{n=0}^{\infty} R^{n} \xi\left(t-n \tau_{r}\right) e^{-i\left(n \Delta t-\Phi_{n}\right)}
$$

where $\Phi_{n}=\Delta \tau_{r} \sum_{l=0}^{n-1} l$, or by the recurrence relation:

$$
E(t)=\xi(t)+R e^{-i \Delta t} E\left(t-\tau_{r}\right) .
$$

Considering that the intracavity laser field is much larger than the spontaneous emission field, the previous expression reduces to: $E(t) \simeq R e^{-i \Delta t} E\left(t-\tau_{r}\right)$. Therefore the time-dependent intensity obeys the relation: $I(t)=E^{*}(t) E(t) \simeq$ $R^{2} I\left(t-\tau_{r}\right)$ which shows that in first approximation the intensity fluctuations of the FSF laser are periodic with the period $\tau_{r}$.

Turning back to the calculation of the coherence functions of the field given in (8) we assume that the roundtrip time in the cavity $\tau_{r}$ is larger than the coherence time $1 / \gamma$ of the stochastic field $\xi(t)$, which ensures the following relation for the two integers $n$ and $p$ :

$$
\left\langle\xi\left(t-n \tau_{r}\right) \xi^{*}\left(t-p \tau_{r}\right)\right\rangle=\delta_{n, p} I_{0}
$$

where $\delta_{i, j}$ is the Kronecker symbol. It follows that for the correlation function $F(\tau)$,

$$
F\left(\tau-n \tau_{r}\right) F\left(\tau-p \tau_{r}\right)=\delta_{n, p} F^{2}\left(\tau-n \tau_{r}\right) .
$$

The time-average degree of first-order coherence of the FSF laser field is identical to the chaotic field:

$$
\overline{g_{F S F}^{(1)}(\tau)}=\frac{\overline{\left\langle E(t) E^{*}(t+\tau)\right\rangle}}{\left\langle E(t) E^{*}(t)\right\rangle}=e^{-i \omega_{0} \tau-\gamma|\tau|} .
$$

The second-order coherence function is found to be independent from the time and writes:

$$
\begin{aligned}
g_{F S F}^{(2)}(\tau) & =\frac{\left\langle E(t) E^{*}(t) E(t+\tau) E^{*}(t+\tau)\right\rangle}{\left\langle E(t) E^{*}(t)\right\rangle^{2}} \\
& =1+\frac{\sum_{p=0}^{\infty} R^{2 p} \sum_{n=0}^{\infty}\left|F\left(\tau-(p-n) \tau_{r}\right)\right|^{2}}{\sum_{p=0}^{\infty} R^{2 p} I_{0}^{2}}
\end{aligned}
$$

The widths of the first- and second-order coherence functions are simply determined by $\gamma$, the spectral width of the chaotic light and are identical to 

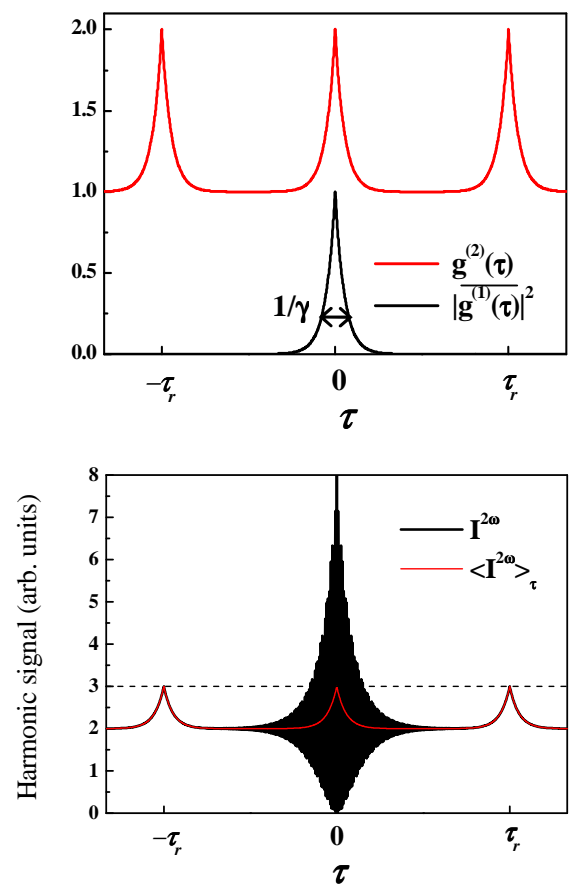

Figure 2: Top: plot of the $\left|g^{(1)}(\tau)\right|^{2}$ and $g^{(2)}(\tau)$ functions for the passive frequency-independent FSF cavity. $R$ is supposed close to 1. Bottom: IAC trace of the FSF light field without (black) and with an average over the fringes (red). 
the case of the LC field. Contrary to the degree of first-order coherence which vanishes outside the coherence domain, the second-order coherence function shows periodic maxima for $\tau=k \tau_{r}$ ( $k$ integer) and $g^{(2)}\left(k \tau_{r}\right)=1+R^{2 k}$ (fig. 2 ). The amplitude of the successive maxima decreases exponentially down to 1 with a characteristic constant $\tau_{0}=1 /(2 \ln R)$, but remains close to 2 provided $R$ is close to 1 . Interestingly this periodic behavior does not depend on the intracavity frequency shift and would also be valid for an unshifted feedback cavity, like a multimode laser. But in that case the first-order coherence function would also be periodic. Therefore the fact that $g^{(1)}(\tau)$ and $g^{(2)}(\tau)$ show a different behavior is the specific signature of FSF lasers, which present photon bunching far beyond the coherence region (fig. 2 top).

We now focus on the IAC signal. The ensemble-average homodyne signal at the output of the interferometer is:

$$
I_{F S F}^{\text {homodyne }}(t, \tau)=1 / 4\left\langle|E(t)+E(t+\tau)|^{2}\right\rangle
$$

which leads to the expression of the time average of the beat signal:

$$
\overline{I_{F S F}^{\text {homodyne }}(\tau)}=\frac{I_{0}}{2\left(1-R^{2}\right)}\left[1+e^{-\gamma|\tau|} \cos \left(\omega_{0} \tau\right)\right] .
$$

The time-dependent intensity of the IAC trace is proportional to:

$$
I_{F S F}^{I A C}(t, \tau)=1 / 16<\left|(E(t)+E(t+\tau))^{2}\right|^{2}>.
$$

Cumbersome but straightforward calculations lead to the following expression for the autocorrelation signal averaged over time:

$$
\begin{aligned}
& I_{F S F}^{I A C}(\tau)=\overline{I_{F S F}^{I A C}(t, \tau)}=\frac{1}{16}\left[\frac{8 I_{0}^{2}}{\left(1-R^{2}\right)^{2}}\right. \\
& +\frac{8 I_{0}^{2}}{1-R^{2}}\left(\frac{F(\tau)}{1-R^{2} e^{i \Delta \tau}}+\frac{F(-\tau)}{1-R^{2} e^{-i \Delta \tau}}\right) \\
& +\frac{2 F(\tau)^{2}}{\left(1-R^{2} e^{i \Delta \tau}\right)^{2}}+\frac{2 F(-\tau)^{2}}{\left(1-R^{2} e^{-i \Delta \tau}\right)^{2}} \\
& \left.+\frac{4}{1-R^{2}} \sum_{p=0}^{\infty} R^{2 p} \sum_{n=0}^{\infty}\left|F\left(\tau-(p-n) \tau_{r}\right)\right|^{2}\right] .
\end{aligned}
$$

Assuming that the frequency shift $\Delta$ is much smaller than $\gamma$ the spectral width of the correlation function, the terms $F( \pm \tau) /\left(1-R^{2} e^{ \pm i \Delta \tau}\right)$ can be approximated by $F( \pm \tau) /\left(1-R^{2}\right)$, which leads to the simpler expression:

$$
\begin{aligned}
& I_{F S F}^{I A C}(\tau)= \\
& \frac{I_{0}^{2}}{4\left(1-R^{2}\right)^{2}}\left[2+4 e^{-\gamma|\tau|} \cos \left(\omega_{0} \tau\right)+e^{-2 \gamma|\tau|} \cos \left(2 \omega_{0} \tau\right)\right.
\end{aligned}
$$




$$
\left.+\left(1-R^{2}\right) \sum_{p=0}^{\infty} R^{2 p} \sum_{n=0}^{\infty}\left|F\left(\tau-(p-n) \tau_{r}\right)\right|^{2}\right] .
$$

The IAC signal exhibits periodic maxima when the path difference equals the cavity roundtrip time (see fig. 2 bottom). As in the case of the chaotic field the ratio of the IAC signal averaged over the fringes at $\tau=0$ to the background outside the coherence domain is $3: 2$. This result is completely equivalent to $g^{(2)}(0)=2$ and is the signature of thermal intensity fluctuations.

The height of the $k^{t h}$ maximum of the IAC trace located at $\tau=k \tau_{r}$ is also found to decrease exponentially as $\left(2+R^{2|k|}\right) / 4\left(1-R^{2}\right)^{2}$.

Finally we turn back to the expression of the homodyne signal. It is worth writing the time-dependent expression of the intensity at the output of the interferometer when the time delay $\tau$ is close to an integer number of the cavity roundtrip time, that is when $\tau=k \tau_{r}+\epsilon$ where $\epsilon \ll \tau_{r}$. Calculations lead to:

$$
\begin{aligned}
& I_{F S F}^{\text {homodyne }}\left(t, k \tau_{r}+\epsilon\right)=1 / 4\left\langle\left|E(t)+E\left(t+k \tau_{r}+\epsilon\right)\right|^{2}\right\rangle \\
& =\frac{I_{0}}{2}\left[\frac{1}{1-R^{2}}+\frac{R^{k} e^{-\gamma|\epsilon|} \cos \left(k \Delta t-\omega_{0} \epsilon+\phi\right)}{\left(1+R^{4}-2 R^{2} \cos \left(2 k \Delta \tau_{r}\right)\right)^{1 / 2}}\right]
\end{aligned}
$$

where $\phi$ is the argument of the complex number $e^{-i \Delta \tau_{r}\left(3 k^{2}-k\right) / 2} /\left(1-R^{2} e^{i 2 k \Delta \tau_{r}}\right)$. The resulting intensity shows a modulation at the angular frequency $k \Delta$ whose amplitude is determined by the value of $2 k \Delta \tau_{r}$. If one assumes $k \Delta \tau_{r}$ is small, which can be realized for instance when $k=1$, then the resulting intensity is:

$$
\begin{aligned}
& I_{F S F}^{\text {homodyne }}\left(t, \tau_{r}+\epsilon\right)= \\
& \frac{I_{0}}{2\left(1-R^{2}\right)}\left[1+R e^{-\gamma|\epsilon|} \cos \left(\Delta t-\omega_{0} \epsilon+\phi\right)\right] .
\end{aligned}
$$

If $\mathrm{R}$ is close to 1 and $\epsilon=0$, the resulting beat intensity is $100 \%$ modulated at the frequency $\Delta$. In other words when the path difference is set equal to a cavity length, the homodyne interferometer performs amplitude modulation of the laser, at the frequency of the frequency-shifter. When the delay equals $k$ times the cavity roundtrip time (k integer), the homodyne signal at the output of the interferometer is modulated at $k \Delta t$. If the laser parameters are adjusted so that $\Delta \tau_{r} / \pi$ is an integer, then the amplitude of the modulation is maximum for any value of $k$ : the contrast of this modulation is $R^{k}$. This configuration can be useful for instance, for the generation of amplitude modulation at a high rate ( $\mathrm{MHz}$ to $\mathrm{GHz}$ range). This point is discussed in the conclusion. When the delay $\tau$ is slightly detuned from $k \tau_{r}(\epsilon \neq 0)$, the amplitude of the RF modulation of the beat signal decreases as $e^{-\gamma|\epsilon|}$ : therefore the homodyne signal exhibits a significant RF beating at $k \Delta$ provided the time difference $\tau-k \tau_{r}$ is kept smaller than the coherence time of the laser. Interestingly this effect shows analogy with white-light interferometry: white-light fringes appear periodically when $\tau=k \tau_{r}$, except that the fringes are shifted from the zero observation frequency to a nonzero frequency equal to $k \Delta$. 


\subsection{Frequency-dependent effective gain}

The simple model of a passive frequency-independent FSF cavity implies that any photon emitted by the gain medium in the spatial mode of the cavity survives with identical probability. It is also possible to include the frequency dependence of gain and losses in the cavity at the expense of more complicated calculations. Authors of [28] showed that when gain and losses are taken into account the width (resp. shape) of the peaks in the intensity correlation function is fixed by the spectral width (resp. shape) of the source. Moreover the amplitude of the successive maxima of $g^{(2)}(\tau)$ decreases according to a Gaussian law $e^{-\left(\tau / \tau_{c}\right)^{2}}$ where $\tau_{c}$ is the effective lifetime of a photon in the cavity. Therefore the coherence and autocorrelation functions of a real FSF laser are expected to follow the same behavior as fig. 2 with the following changes: the shape of the peaks is not Lorentzian but Gaussian (for a Gaussian output spectrum) with a width equal to the coherence length of the source, and the height of the peaks decreases with $\tau$ according to a gaussian law, compared to the exponential decay obtained for the passive frequency-independent FSF cavity. But the ratio of the height of the central autocorrelation peak over the background is still 3:2, which corresponds to $g^{(2)}(0)=2$.

\section{Experimental results}

\subsection{Experimental setup}

In this part we present the experimental demonstration of the previous theoretical predictions by measuring the homodyne signal and the IAC trace of a CW dye FSF laser at the output of a Michelson interferometer enabling large path difference. In the frame of LGS applications we developed a FSF dye ("modeless") laser tunable around $589 \mathrm{~nm}$ [7]. A CW 3W frequency-doubled Nd:YAG laser pumps the Rhodamine $6 \mathrm{G}$ jet of a commercial L-shaped laser cavity. The free spectral range is $283 \mathrm{MHz}$, corresponding to a cavity length $l_{c}=530 \mathrm{~mm}$. An acousto-optics modulator operating at $40 \mathrm{MHz}$ is inserted in the cavity $\left(\Delta=2 \times 2 \pi \times 40.10^{6} \mathrm{rad} / \mathrm{s}\right)$. The latter is closed on the +1 diffraction order. Without additional selective elements in the cavity, the output spectrum is measured and shows a $85 \mathrm{GHz}$ FWHM. We also have the possibility to insert a Fabry-Perot etalon to narrow the spectral width down to $3 \mathrm{GHz}$ in the perspective of exciting sodium D transitions for the LGS applications. (It is worth to note that in both cases the time scale of the laser intensity fluctuations is in the ps range and would require very fast detection to be evidenced). The output intensity is about $200 \mathrm{~mW}$. The laser beam feeds a Michelson interferometer where the path difference between both arms can be as large as $1.20 \mathrm{~m}$, i.e. larger than twice the length of the laser cavity (fig. 3). The interferometer is mechanically scanned at a typical average speed of $10 \mathrm{~cm}$-path difference per second. At the output of the interferometer, the beam is focused onto a type I BBO crystal whose angle is adjusted to the phase-matching condition for the second-harmonic generation of the beam at $589 \mathrm{~nm}$. A dichroic mirror enables 


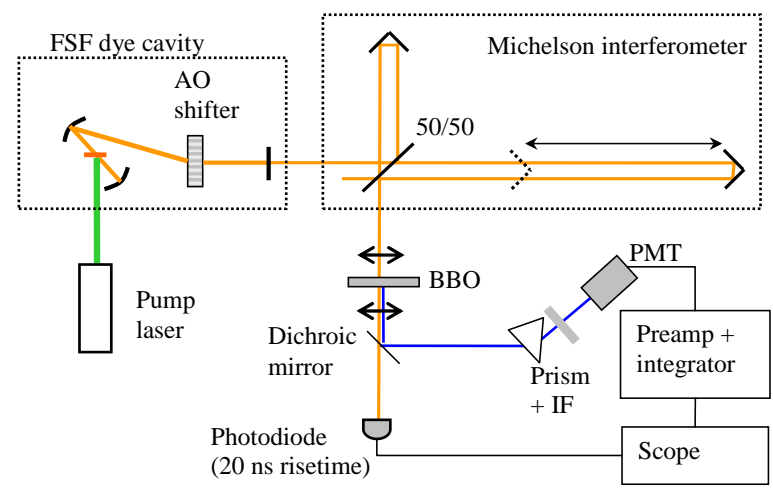

Figure 3: Experimental setup (see text for details).

to separate the harmonic component at $294.5 \mathrm{~nm}$ from the fundamental one. The latter is detected by a photodiode with a $20 \mathrm{~ns}$ rise time. The harmonic signal is filtered by means of a dispersive prism and a $10 \mathrm{~nm}$ bandwidth interference filter (IF), and detected by a PM tube. The output current is preamplified and averaged by a $4 \mathrm{~ms}$ time constant integrator. A digital oscilloscope with a long capture time $(1 \mathrm{Gs} / \mathrm{s})$ enables to record long $(10 \mathrm{~s})$ scans. The RF spectrum of the beat signal for a given value of the path difference is also calculated by the same oscilloscope using a FFT procedure.

\subsection{RF spectrum of the beat signal}

First we study the variation of the RF spectrum of the laser beam after the interferometer, as a function of the path difference (fig. 4, top). A strong beat at the free spectral range $(283 \mathrm{MHz})$ is always recorded, corresponding to the periodic repetition of the intrinsic fluctuations of the stochastic spontaneous emission. We also observe that the multiples of the AO frequency are always present inducing additional intensity noise in the RF spectrum due to the AO modulation. The usual satellite components used in metrology are recorded and their position evolve linearly with the path difference with a rate $\Delta /\left(4 \pi l_{c}\right)$. When the path difference is equal to $2 l_{c}$, the first satellite is located at $\Delta /(2 \pi)$ and its amplitude increases dramatically. Then the beat signal monitored by a fast photodiode (1 ns risetime) exhibits a near $100 \%$ amplitude modulation with a period of $12.5 \mathrm{~ns}(1 / 80 \mathrm{MHz})$ as expected from section 1 (fig. 4, bottom).

\subsection{Interferometric autocorrelation of the FSF laser}

We now turn to the measurement of the autocorrelation trace of the FSF laser. The interferometer is scanned and the homodyne and IAC signals are recorded and plotted as a function of the path difference (fig. 5 and 6 ). In the vicinity of 


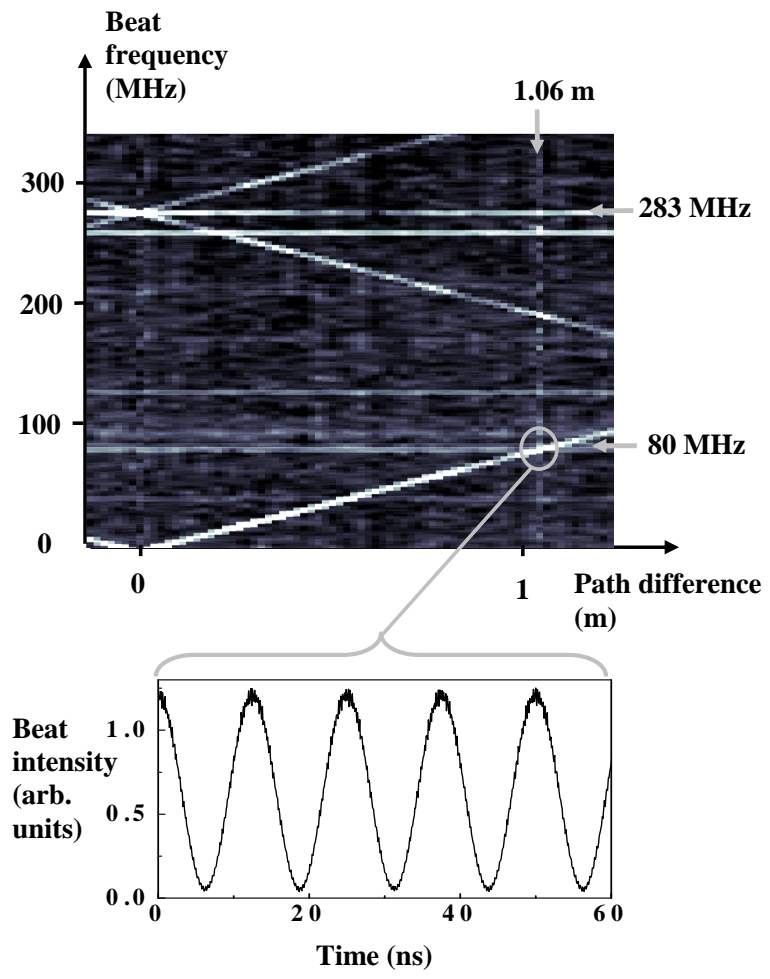

Figure 4: Top: experimental RF spectrum of the homodyne signal with respect to the path difference in the interferometer. The RF peak at $250 \mathrm{MHz}$ is an electronic parasit. Bottom: homodyne signal at the output of the interferometer when the path length is set at $1.06 \mathrm{~m}$. Detection made use of a $1 \mathrm{~ns}$ risetime photodiode. Fast intensity fluctuations (ps range) are washed out by the dynamics of the detection. 

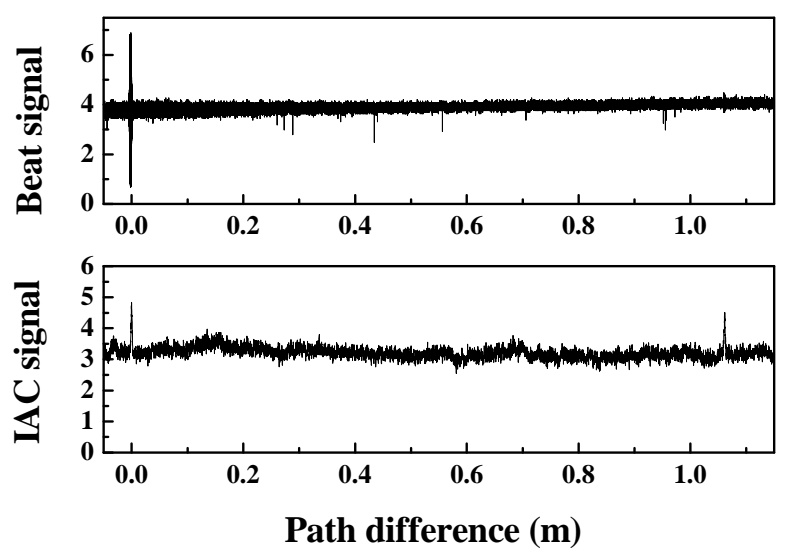

Figure 5: Variation of the homodyne and autocorrelation signals with the path difference over a full scan of the interferometer. Peaks on the IAC trace are clearly visible at a path difference equal to 0 and $1.06 \mathrm{~m}$.

null path difference the usual interferogram is recorded in the homodyne signal (fig. 6 (a)). The IAC trace averaged over the fringes by the integrator, exhibits a maximum, illustrating large intensity fluctuations or photon bunching (fig. 6 (b)). The ratio between the peak height and the background is around 1.45, i.e. slightly smaller than the expected value of 1.5. This small discrepancy is probably partly due to the fact that the interferometer is slightly imperfectly balanced as illustrated by the fact that the contrast of the interference fringes is not exactly $100 \%$.

In the vicinity of $1.06 \mathrm{~m}$, the path difference of the interferometer is close to twice the cavity length. Therefore the IAC trace corresponds to the correlation of the FSF field with the field emitted one cavity roundtrip later. The homodyne signal shows no fringes, which corresponds to the fact that both fields are not first-order mutually coherent: indeed in first approximation they are shifted by $80 \mathrm{MHz}$. However the IAC trace exhibits the expected maximum, illustrating the second-order mutual coherence of the fields. The ratio between the maximum and the background on the autocorrelation signal is close to 1.4, partly due to the slight lack of balance of the interferometer and also to the fact that after a $1 \mathrm{~m}$ path difference, the spatial modes of both fields are slightly different which decreases the relative contrast of the SHG signal.

\section{Conclusion and perspectives}

The statistical properties of FSF lasers have been investigated both theoretically following the treatment given in [28], and experimentally by means of interferometric autocorrelation measurements. Our data confirm the chaotic nature 

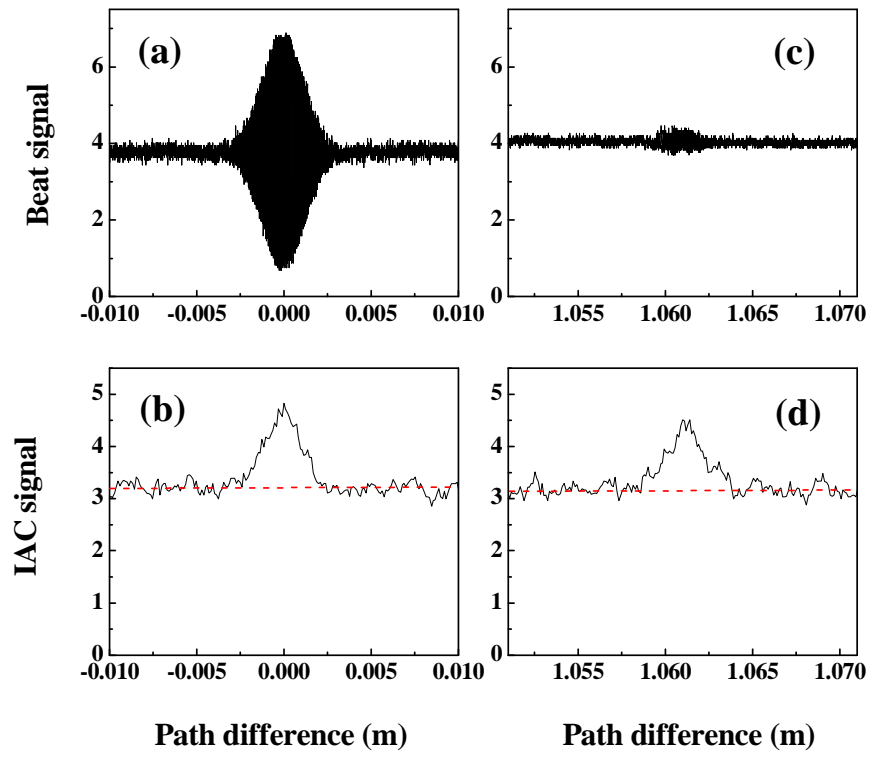

Figure 6: Evolution of the homodyne (top) and autocorrelation signals (bottom) when the path difference is close to 0 (left) and to $1.06 \mathrm{~m}$ (right) for the same scan as fig. 5. Top right: the small modulation of the homodyne signal in the vicinity of $1.06 \mathrm{~m}$ is an artefact coming from the fact that the resulting homodyne signal is modulated at $80 \mathrm{MHz}$, that is slightly too slow to be completely averaged by the $20 \mathrm{~ns}$ risetime photodiode. 
of the output field of FSF lasers and are in agreement with the description of the output field as a periodic sequence of spontaneous emission events, periodically shifted in frequency. A FSF laser is a cyclostationary thermal light source exhibiting strong intensity fluctuations or photon bunching. Interestingly, the second-order coherence function of FSF laser also shows periodic maxima, contrary to the first-order coherence function whose variations are localized within the coherence length of the laser. A first possible application of this peculiar laser source could be the realization of very high frequency amplitude modulation (AM). If one recombines the output FSF laser field with the field emitted $k \tau_{r}$ later ( $k$ is an integer), the resulting light field is modulated at the angular frequency $k \Delta$. The amplitude of the modulation depends on the cavity parameters but can be close to $100 \%$. The other condition for strong modulation is that $k \tau_{r}$, the time difference between both paths should be smaller than $\tau_{c}$ the average time a photon spends in the cavity, otherwise the memory of the laser field is lost and the fields $E(t)$ and $E\left(t+k \tau_{r}\right)$ loose their relative second-order coherence, even if very high orders $(k \sim 20000)$ beatings have been evidenced [11]. But one can expect to generate AM in the GHz range provided the spectral width of the laser is large enough. Among others such a source might find applications in atomic physics, where coherent population trapping or electromagnetically induced transparency schemes require to excite coherently two transitions at different frequencies. Another field of applications could be lidar radar techniques where RF radar signals are carried by an optical carrier wave [38]. Finally we also believe FSF lasers could find applications in the growing field of quantum imaging, which is based on the measurement of intensity correlations [39]. Thermal light sources have recently demonstrated their po-

tential compared to twin photon sources and FSF lasers displaying thermal and periodic fluctuations could constitute interesting devices for this emerging field.

\section{References}

[1] F. V. Kowalski, P. D. Hale, S. J. Shattil, Opt. Lett. 13 (1988) 622.

[2] F. V. Kowalski, S. J. Shattil, P. D. Hale, Appl. Phys. Lett. 53 (1988) 734.

[3] L. P. Yatsenko, B. W. Shore, K. Bergmann, Opt. Comm. 236 (2004) 183.

[4] I. C. M. Littler, K. Bergmann, Opt. Comm. 88 (1992) 523.

[5] M. J. Lim, C. I. Sukenik, T. H. Stievater, P. H. Bucksbaum, R. S. Conti, Opt. Comm. 147 (1998) 99.

[6] H. Guillet de Chatellus, J.-P. Pique, Opt. Lett. 34 (2009) 755.

[7] J.-P. Pique, S. Farinotti, J. Opt. Soc. Am. B 20 (2003) 2093.

[8] H. Guillet de Chatellus, J.-P. Pique, I. C. Moldovan, J. Opt. Soc. Am. A 25 (2008) 400. 
[9] I. C. M. Littler, H.-M. Keller, U. Gaubatz, K. Bergmann, Z. Phys. D 18 (1991) 307.

[10] D. T. Mugglin, A. D. Streater, S. Balle, K. Bergman, Opt. Comm. 104 (1993) 165.

[11] K. Nakamura, T. Hara, M. Yoshida, T. Miyahara, H. Ito, IEEE J. Quant. Elec. 36 (2000) 305.

[12] V. V. Ogurtsov, L. P. Yatsenko, V. M. Khodakovskyy, B. W. Shore, G. Bonnet, K. Bergmann, Opt. Comm. 266 (2006) 266.

[13] S. Reza, R. Ricken, V. Quiring, W. Sohler, EPS-QEOD Europhoton Conference, Pisa/Italy (2006).

[14] M. Yoshida, T. Miyamoto, N. Zou, K. Nakamura, H. Ito, Opt. Exp. 9 (2001) 207.

[15] S. Umemoto, K. Kubota, Y. Fujino, T. Hara, N. Miyamoto, T. Okamoto, International Conference on Structural Health Monitoring of Intelligent Infrastructure, Vancouver/Canada (2007).

[16] N. Kibayashi, T. Hara, M. Yoshida, K. Nakamura, H. Ito, Proc. SPIE 4150 (2001).

[17] F. V. Kowalski, C. Ndiaye K. Nakamura, H. Ito, Opt. Comm. 231 (2004) 149.

[18] I. A. C. Littler, J. H. Eschner, Opt. Comm. 87 (1992) 44.

[19] G. Bonnet, S. Balle, T. Kraft, K. Bergmann, Opt. Comm. 123 (1996) 790.

[20] S. Balle, K. Bergmann, Opt. Comm. 116 (1995) 136.

[21] M. Stellpflug, G. Bonnet, B. W. Shore, K. Bergmann, Opt. Exp. 11 (2003) 2060 .

[22] S. Balle, I. C. M. Littler, K. Bergmann, F. V. Kowalsi, Opt. Comm. 102 (1993) 166.

[23] N. Nakamura, K. Kasahara, M. Sato, H. Ito, Opt. Comm. 121 (1995) 137.

[24] F. V. Kowalski, N. Nakamura, H. Ito, Opt. Comm. 147 (1998) 103.

[25] K. Kasahara, K. Nakamura, H. Ito, IEEE J. Quant. Elec. 34 (1998) 190.

[26] K. Nakamura, T. Miyahara, H. Ito, Appl. Phys. Lett. 72 (1998) 2631.

[27] A. Yoshizawa, H. Tsuchida, Opt. Comm. 155 (1998) 51.

[28] L. P. Yatsenko, B. W. Shore, K. Bergmann, Opt. Comm. 282 (2009) 300.

[29] I. A. C. Littler, S. Balle, K. Bergmann, J. Opt. Soc. Am. B 8 (1991) 1412. 
[30] F. V. Kowalski, C. Ndiaye, K. Nakamura, H. Ito, Opt Lett. 27 (2002) 1965.

[31] J. Paul, Y. Hong, P. S. Spencer, K. A. Shore, Proc. SPIE 6909 (2008).

[32] F. T. Arrechi, Phys. Rev. Lett. 15 (1965) 912.

[33] M. C. Teich, G. J. Wolga, Phys. Rev. Lett. 16 (1966) 625.

[34] Y. Qu, S. Singh, Opt. Comm. 90 (1992) 111.

[35] H. P. Weber, H. G. Danielmeyer, Phys. Rev. A 2 (1970) 2074.

[36] K. L. Sala, G. A. Kenney-Wallace, G. E. Hall, IEEE J. Quant. Elec. 16 (1980) 990.

[37] R. Loudon, "Quantum Theory of Light", 3rd ed. (Oxford U. Press, New York, 2000), Chap. 6.

[38] L. Morvan, N. D. Lai, D. Dolfi, J.-P. Huignard, M. Brunel, F. Bretenaker, A. Le Floch, Appl. Opt. 41 (2002) 5702.

[39] Y. Shih, IEEE J. Sel. Top. in Quant. Elec. 13 (2007) 1016. 